DOI: 10.12731/2227-930X-2017-1-38-51

\title{
MODELLING OF DYNAMIC SPEED LIMITS USING THE MODEL PREDICTIVE CONTROL
}

\author{
Nikolaev A.B., Myo Min Khaing, Aung Myo Thwin, \\ Moe Ko Ko, Myo Lin Aung
}

The article considers the issues of traffic management using intelligent system "Car-Road" (IVHS), which consist of interacting intelligent vehicles (IV) and intelligent roadside controllers. Vehicles are organized in convoy with small distances between them. All vehicles are assumed to be fully automated (throttle control, braking, steering). Proposed approaches for determining speed limits for traffic cars on the motorway using a model predictive control (MPC). The article proposes an approach to dynamic speed limit to minimize the downtime of vehicles in traffic.

Keywords: intelligent transport system; intelligent car; simulation; traffic safety; model MPC; control in IVHS; speed limit.

\section{Introduction}

The ever increasing demand for mobility leads to traffic jams on the roads in worldwide. One of the most promising approaches to reduce the frequency and impact of congestion is the use of advanced traffic management methods that control measures (traffic lights, dynamic route information displays, dynamic speed limits, etc.) are used to control the flow of vehicles in order to reduce traffic congestion and improve highways crossing abilities.

Modern traffic control technology based on the modern transport infrastructure based on the use of information and communication technologies, including intelligent equipment in cars. In road transport, such systems are called intelligent vehicle highway system (IVHS). All vehicles are equipped with additional IVHS equipment which allows them to move in groups with a distance of two meters between the vehicles and the distance up to 30-60 meters between such groups. High 
speed and short distances between vehicles in the group can increase the capacity of the motorway. The proposed approach does not ignore the usual traffic control measures. In this paper, we will consider how to determine the appropriate speed limits for vehicles in groups IVHS to optimize the performance of traffic system, to reduce travel time and fuel consumption, improve security and reliability.

\section{The hierarchical framework for management in IVHS}

The hierarchical traffic management framework in IVHS (Figure 1) provides increased throughput and reduces the risk of traffic jams, and contains several levels:

- High-level controllers (regional, supraregional and district administration) ensure coordination of the upper levels of management.

- Roadside controllers. Each group of vehicles in the road network is considered the roadside controller as a single unit. This greatly reduces the complexity of managing the problem, compared with a case where every single vehicle will be controlled by roadside controller. As a result, all network traffic can be managed more efficiently.

- Groups of vehicles controllers - receive commands from the roadside controllers, and are responsible for the oversight and coordination of each car group within the vehicles. They provide the coordinated motion of group of vehicles, including maneuvers and maintaining safe distances between vehicles in the group.

- Vehicle Controllers - receive commands from groups of vehicles controllers and translate these commands into control signals for actuators, which installed in every vehicles (throttle, braking, steering).

The main objective of the roadside controller is to determine the speed of movement of the group of vehicles and the safe distance to avoid collisions between vehicles, to determine the best size of the group of vehicles according to the specific traffic conditions, dynamic routing for groups of vehicles and output commands to change the number and size of the group of vehicles. 


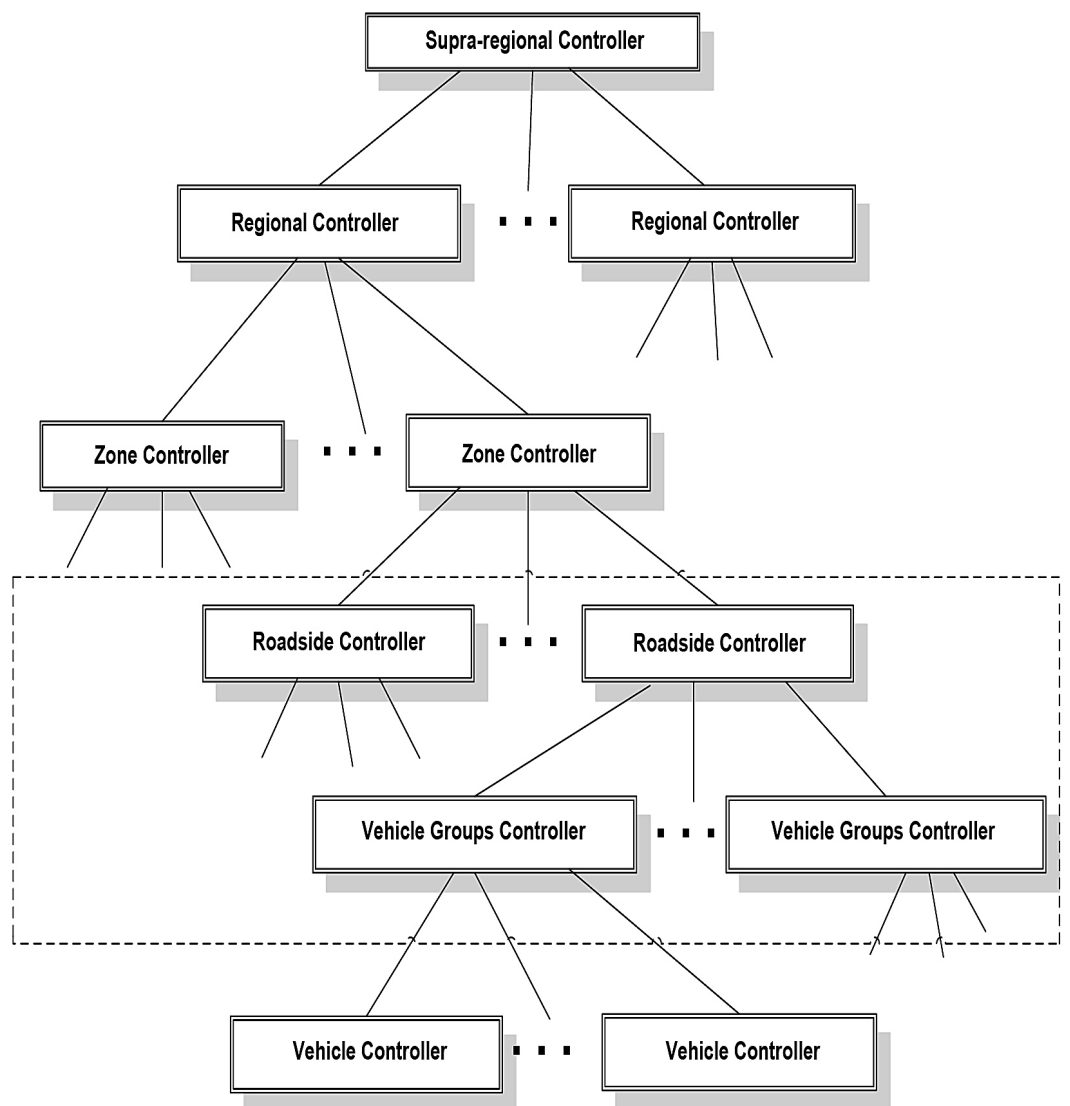

Fig. 1. The hierarchical management structure IVHS

\section{Model predictive control for traffic management using IV}

Model predictive control (MPC) has originated in the process industry and has been successfully implemented in many other industries. MPC uses a model with discrete time $T_{c t r l}$. At each control step, MPC controller determines the current state $x(k)$ of the system. Next, using an explicit prediction model determined the values of the control commands for a prediction determined period of $N_{p}$ (Figure 2). 


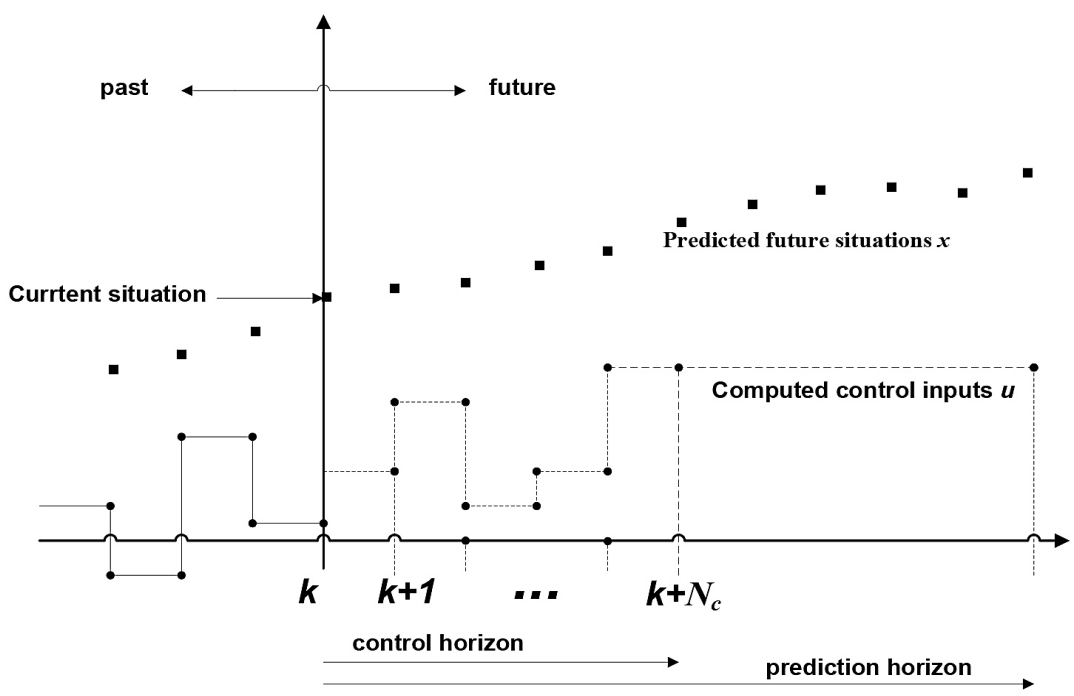

Fig. 2. Prediction Management horizon in MPC

In order to reduce the computational complexity of the problem, often impose the restrictions in the form of $u(k+j)=(k+j-1)$, for $j=N_{c}, .,{ }_{,} N_{p}-1$ where $N_{c}$ is called the prediction horizon. In each control step, the control command generated by $u^{*}(k)$, which is an element of optimal control sequence $u *(k), \ldots, u *\left(k+N_{c}-1\right)$. Further, the prediction horizon is shifted one step forward, and forecasting process is repeated using the newly obtained values. Consider the possibility to control the speed within the IVHS using MPC.

Roadside controller works with groups of vehicles as basic entities. In this case, the control signal must be set the speed limit for the main vehicle in the group, and the time of travel controller. Today, there is a wide range of traffic patterns in the area. An important factor that determines the selection of models for use in the MPC is a compromise between accuracy and computational complexity, since at each control step $\mathrm{k}$ the model will be used repeatedly in real time during the entire running time. As a consequence, very detailed (microscopic) simulation model of the motion, as a rule, are not suita- 
ble as a predictive model of MPC. Instead, usually used simplified (aggregated) model. However, it should be noted that the MPC is constructed in a modular fashion. In that case, the model predicting inadequate computational efficiency, it can easily be replaced by another predictive model. As a performance criteria $J_{\text {perf }}(k)$ for MPC for IVHS are the total time, overall throughput, the total fuel consumption, safety, or combination of these, evaluated for the time period $\left[k T_{c t r p}\left(k+N_{p}\right) T_{c t r r}\right)$.

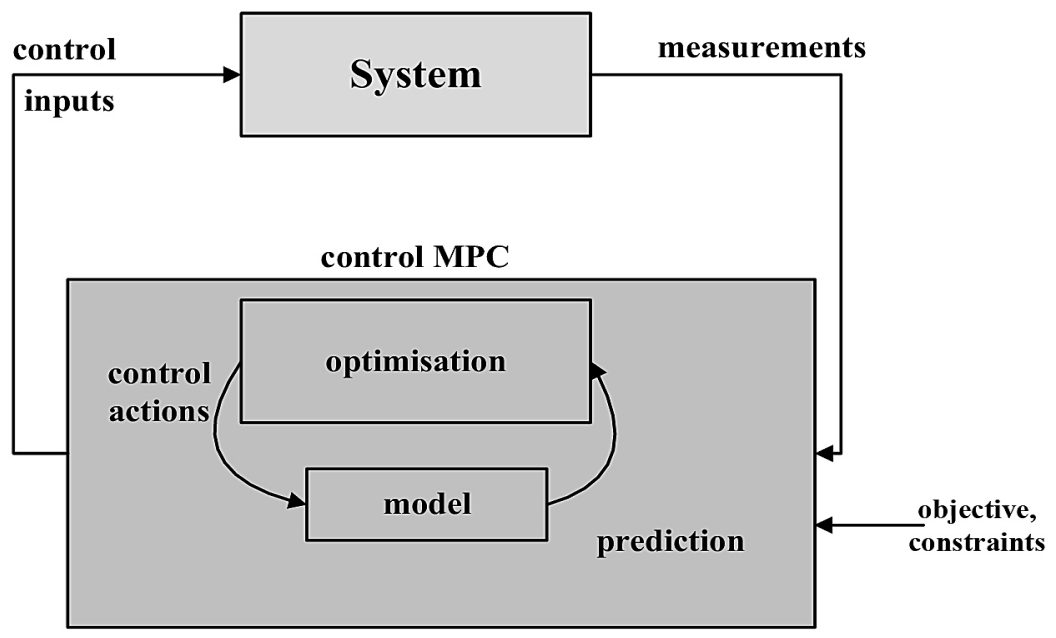

Fig. 3. The control structure MPC

In order to prevent the traffic pulsation in the control signals is often added a penalty on variations in the control signal $u$, which leads to increased productivity of complexity functions:

$$
J_{\text {tot }}(k)=J_{\text {perf }}(k)+\alpha \sum_{j=0}^{N_{c}-1}\|u(k+j)-u(k+j-1)\| 2
$$

the control step $k$, where $\alpha>0$ is a weighting factor.

MPC controller can also explicitly take into account the operational constraints such as the minimum distance between the groups of vehicles and maximum speed, minimum advance, etc. 


\section{Components of models IVHS}

This section describes a simplified model of vehicle and groups of vehicles, which can be used as a predictive model in a roadside controller using MPC. In the absence IVHS, vehicles can move at any speed within a permitted speed (e.g. $120 \mathrm{~km} / \mathrm{h}$ ). As the traffic demand increases, the vehicles start to follow their predecessors at closer distances and at reduced speeds $(50-80 \mathrm{~km} / \mathrm{h})$. When the capacity of the highway is being utilized at its maximum, decreases speed to $0-40 \mathrm{~km} / \mathrm{h}$.

\subsection{Vehicle Model}

To simulate the dynamics of vehicle movement using the equation:

$$
\begin{gathered}
x_{i}(\ell+1)=x_{i}(\ell)+v_{i}(\ell) T_{\text {sim }}+0.5 a_{i}(\ell) T_{\text {sim }}^{2} \\
v_{i}(\ell+1)=v_{i}(\ell)+a_{i}(\ell) T_{\text {sim }}
\end{gathered}
$$

where the $\ell$ - simulating step number, $T_{\text {sim }}$ - simulation time step, $x_{i}(\ell)$ - coordinate the longitudinal position of the vehicle $(i)$ for the time $t=\ell T_{\text {sim }}, v_{i}(\ell)$ - speed of the vehicle $(i)$ for the time $t=\ell T_{\text {sim }}$ and $a_{i}(\ell)-$ acceleration of transport equipment (i) at time $t=\ell T_{\text {sim }}$. Acceleration is present in (2)-(3) and is calculated in accordance with the current driving situation, as will be explained below. Furthermore, the acceleration is limited between the maximum acceleration value $a_{\text {acc,max }}$, and the maximum value of acceleration comfortable deceleration $a_{\text {dec,max }}$.

\subsection{Model for Driver}

In that case, if the of group of vehicle reach the next ahead exceeds than the critical value, it is considered that the vehicle moves independently, rather than as a group. Otherwise, it is assumed that the moves of vehicles in the group. As described, there are various types of vehicle models in the following [5]: a model to stimulate the response [6], the model of collision avoidance [7], psychophysical model [8], and models of cellular automata [9]. We use the model to stimulate the response [6] to describe the behavior of the driver, since this model is 
the most commonly used and easy to implement. Model stimulus response [11] is based on the hypothesis that each vehicle is accelerated or decelerated in accordance with the relative velocity and the distance between the vehicles and its predecessor. Then, acceleration is determined by the formula [11]:

$$
a_{i}(\ell)=C v_{i}^{\beta}(\ell) \frac{\left(v_{i+1}(\ell-d)-v_{i}(\ell-d)\right)}{\left(x_{i+1}(\ell-d)-x_{i}(\ell-d)\right)^{\gamma}}
$$

where $C, \beta$ and $\gamma$ are parameters of the model, and $d$ is the delay of the driver.

(1-Assume that $T_{\text {react }}$ - reaction time, which typically has a value of 1-1.2s, is an integer multiple of simulation time step Tsim. So, $T_{\text {react }}=\sigma T_{\text {sim }}$ where $\sigma$ - Integer.

2 -It is assumed that $T_{\text {delay }}$ usually has a value of from 1-1.2 $\mathrm{s}$, and is an integer multiple of $T_{\text {sim. }}$. Thus $T_{\text {delay }}=d T_{\text {sim }}$, where d-Integer).

\subsection{Models for intelligent vehicles}

Intelligent Vehicle (IA) used adaptive cruise control (ACC) and intelligent speed adaptation (ISA) when driving in the part of the group of vehicles. Consider the model for calculating the acceleration of the head of the groups of vehicles and other vehicles in the group.

1) model for the head of the group of vehicles: the calculation of the acceleration is made on the basis of the expression:

$$
a_{i}(\ell)=K_{1}\left(v_{I S A}(\ell)-v_{i}(\ell)\right)
$$

where $K_{l}$ is - the constant of proportionality, $v_{I A S}$ - initial rate value, which is measured by the roadside controller.

2) model for other vehicles: in this case, the acceleration is calculated based on:

$$
a_{i}(\ell)=K_{2}\left(h_{\text {init }, i}(\ell)-\left(x_{i+1}(\ell)-x_{i}(\ell)\right)\right)+K_{3}\left(v_{i+1}(\ell)-v_{i}(\ell)\right)
$$

where $K_{2}$ and $K_{3}$ - constants and $h_{\text {init, } i}$ - distance to advance the starting of the car $(i)$. The controller calculates a safe distance forward as follows: 


$$
h_{\text {init }, i}(\ell)=S_{0}+v_{i}(\ell) T_{\text {ford }, i}+L_{i}
$$

where $S_{0}$ - the minimum safety distance to be maintained at zero speed. $T_{\text {ford }, i}-$ Time to move forward for the vehicle $(i)$ and $L_{i}-$ the length of the vehicle $(i)$.

\subsection{Model for the Group of IV}

On a more general level, one can also consider a group of vehicles, as a single entity without regard to the detailed description of the interaction between the individual vehicles in the group. So essentially, we consider a platoon as one vehicle with a length that is a function of the speed of the platoon (due to the dependence of the intervehicle spacing managed by the ACC on the speed and the number and length of vehicles in the group). Consider p group and assume for simplicity that the vehicle group are numbered from 1 (the last car group), 2 (the penultimate group of vehicles), ...., $n_{p}$ (group leader). The speed depends on the length of the group:

$$
L_{\text {group }, p}=\left(n_{p}-1\right)\left(S_{0}+S_{1} v_{n_{p}}(\ell)\right)+\sum_{i=1}^{n_{p}} L_{i}
$$

where $S_{0}+S_{1} v_{n_{p}}(\ell)$ - the speed depends on the distance between the vehicle in the group, the $S_{1}$ constant, and $v_{n_{p}}$ is the speed of cars (leader).

When merging the groups of vehicles can use the group model, if the merger will not be lead to a collision at the following times intervals. If both conditions are satisfied, the group of the vehicles is joined to the main flow (at a rate, which is determined by roadside controller).

For individual vehicles not controlled by the roadside controller can use the similar model, i.e., the vehicle joins the mainstream line provided that there is a large enough distance between the groups, and that collision will not occur. The vehicle speed can be taken equal to the speed of the vehicle in front or a value not exceeding the maximum permitted speed.

\section{Example simulation}

To illustrate the operation of IVHS, consider the segment of the road length of $13 \mathrm{~km}$. At a distance of $7.5 \mathrm{~km}$, is provided a branch from the beginning of this section (Figure 4). 


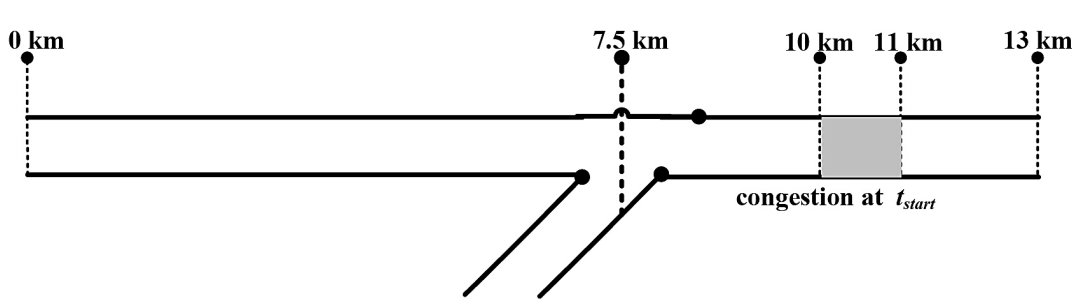

Fig. 4. Fragment of the road

Let us consider the motion of vehicles without IVHS, with speed (ISA) and the movement of intellectual adaptation of IA system. For simplicity, all the vehicles are considered to be of the same length $\left(L_{i}=4 \mathrm{~m}\right)$. During the simulation, we believe that the ISA limits the speed of the vehicle and that drivers may not exceed the imposed speed limit. In the IV-based case the groups of vehicles. Suppose that all the IVs equipped with all necessary facilities (computers, sensors, and ACC and ISA controllers on board).

Modeling a period of 9 minutes starting from the time $t_{\text {start }}=7$ hours, ending at time $t_{\text {end }}=7 \mathrm{~h} 9$ mins. The intensity of the movement of vehicles assumed to be constant during the simulation period, and 1365 $\mathrm{veh} / \mathrm{h}$ for non-automated vehicles, and $404 \mathrm{veh} / \mathrm{h}$ for the agency.

In the present state of the initial state of the simulation scenario is described as follows. There is congestion of vehicles on the segment from $x=10 \mathrm{~km}$. to $x=11 \mathrm{~km}$. at time $t_{\text {start }}$. In congested area there are 100 vehicles at a speed of $0 \mathrm{~km} / \mathrm{h}$. The area has not overloaded vehicles 70 (uniformly distributed) at a speed of $120 \mathrm{~km} / \mathrm{h}$. After time $t_{\text {start }}$, the traffic congestion in the area returns slowly to its normal value.

For the research model was developed in the Matlab environment, simulated and compared the results obtained for this scenario in the normal driving mode and using the IVHS. Model used to model vehicle (2)-(3) with the relative acceleration. To calculate the relative acceleration during normal driving of the equation (4). For groups of cars with the ISA - the equations (5)-(7). For the head of the car in the group $C=1,55, \beta=1,08$ and $\gamma=1,65$ to accelerate, and $C=2,55, \beta=-1,67$ and $\gamma=-0,89$ for braking. Moreover, selected $\sigma=1, d=1, K=0.01$ 
and $K_{1}=0,4$. For all vehicles in the group we have $K_{2}=0,6, K_{3}=1.2$, $S_{0}=3$ and $T_{\text {ford }}=0.5$. For a group of vehicles in a model (8) is chosen $S_{1}=0.5$. Furthermore, $a_{\text {acc, } \max }=3$ and $a_{\text {dec, } \max }=-3$ for all models. Time step simulation made $T_{\text {sim }}=1 \mathrm{~s}$.

The purpose of traffic management is to increase the traffic performance, i.e. minimizing the total time spent (TTS) for all vehicles using dynamic speed limits:

$$
J_{T T S, \text { sim }}=\sum_{\ell=1}^{N_{\text {sim }}}\left(n_{\text {veh }}(\ell)\right)+q_{\text {highway }}(\ell)+q_{\text {on-ramp }}(\ell) T_{\text {sim }},
$$

where $N_{\text {sim }}=540$, the total number of simulation steps (length $T$ sim $=$ 1s) during the entire period of simulation 9 minutes $n_{v e h}(\ell)$ is the number of vehicles that are present in the network at time $t=t_{\text {start }}+\ell T_{\text {sim }}$, $q_{\text {highway }}(\ell)$ is the number of cars in the queue for travel on the highway during $t=t_{\text {start }}+\ell T_{\text {sim }}$ and $q_{\text {on-ramp }}(\ell)$ - the number of vehicles present in the queue at time $t=t_{\text {start }}+\ell T_{\text {sim }}$.

The corresponding function efficiency $J_{\text {perf }}(k)$, when used in the MPC step approach to control is then given,

$J_{\text {perf }}(k)=\sum_{\ell=k K+1}^{\left(k+N_{p}\right) K}\left(n_{v e h}(\ell)+q_{\text {highway }}(\ell)+q_{\text {on-ramp }}(\ell)\right) T_{\text {sim }},(10)$ with $K=\frac{T_{c t r l}}{T_{\text {sim }}}$. The total target MPC functions are also included, the term fine $\alpha=0,01$. For a control of the human situation, the ISA Management measures applied (with a speed limit for each section of $1 \mathrm{~km}$ between the position of $x=0 \mathrm{~km}$ and the position $x=10 \mathrm{~km})$. So, $u$ for the MPC control signal of the control task to step $k$ includes ISA speed limit for the first 10 sections and measuring speeds (expressed as a number between 0 and 1) control step $k$ to $k+N_{c}-1$ (i.e., $11 N_{c}$ variables in total).

If $P_{k}$ amount present in the network vehicle groups at the control point $\mathrm{k}$, and if $Q_{k}$ is the number of cars groups which could enter into the network during the time interval from $t=k T_{c t r l}$ to $t=\left(k+N_{p}\right) T_{c t r l}$, the total number of variables is determined $P_{k} N_{c}+Q_{k}$ in total. 
Consider a maximum speed of $120 \mathrm{~km} / \mathrm{h}$. Roadside controller must ensure the maintenance of a safe distance between cars in the group, and between groups of cars. In particular, the minimum safe distance between the group cars $p_{1}$ and following the same lane in front of a group of $p_{2}$ cars is set $S_{0^{\prime} \text { group }+} T_{\text {ford, group }} v_{\text {gruop }} p 1$ where $v_{\text {gruop }, p 1}$ has a cars group speed $p_{1}$. For the case study $S_{0^{\prime} \text { group }}=20 \mathrm{~m}$ and $T_{\text {ford, group }}=2 \mathrm{~s}$.

The access time $T_{c t r l}$ control is set to $1 \mathrm{~min}$. For prediction horizon $N_{p}$ was made a value that matches $7 \mathrm{~min}$, and is set to control horizon $N_{c}$, which corresponds to 3 minutes in order to limit the number of variables.

\section{Results}

The simulation results showed a significant reduction in the total time spent (TTS) in the case of the control groups of vehicles. Compared to the uncontrolled movement of the improvement was more than $24 \%$ (Table 1 ).

Table 1.

Values of the total time spent on the network during the simulation period

\begin{tabular}{|l|c|c|}
\hline \multicolumn{1}{|c|}{ Case } & TTS(veh/h) & Relative improvement \\
\hline Uncontrolled case & 27.44 & $0 \%$ \\
\hline Controlled ( human drivers) & 24.72 & $9.91 \%$ \\
\hline Controlled (car groups) & 20.67 & $24.67 \%$ \\
\hline
\end{tabular}

These results can be explained as follows. In the uncontrolled case, when there are no vehicle in front of the driver or, if there is enough space between vehicles, drivers retain their desired speed. But when the driver is faced with traffic jams, he has to brake to avoid a collision, and he had to wait until this incident will cleared. Moreover, there are no mechanisms that can delay or prevent the flow of additional vehicles from entering the highway. All this results leads to a lot of time spent in the network for the vehicle, and thus also a higher value TTS (total time spent). 


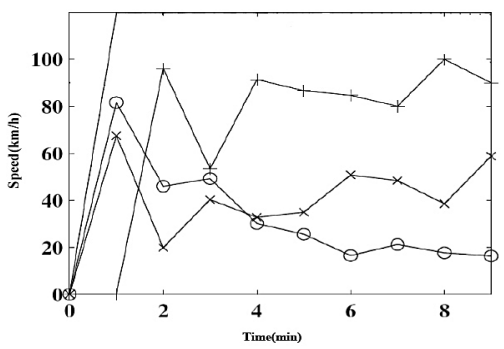

(a) simulation

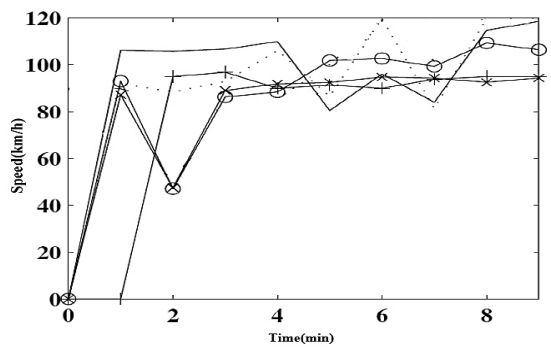

(b) simulation for controlled (human drivers) for uncontrolled case

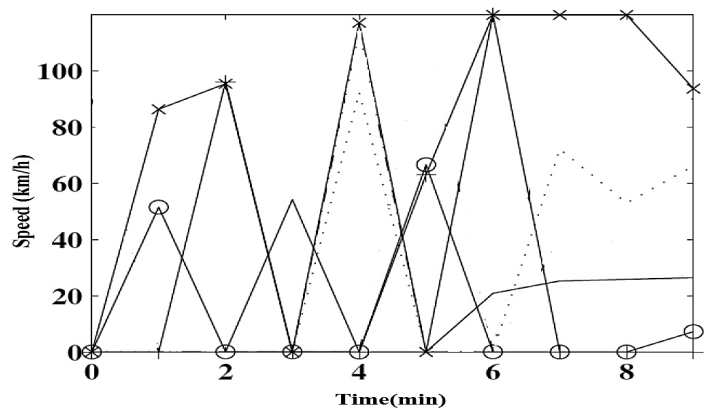

(c) simulation for controlled (car groups)

Fig. 5. Simulation graphs of results

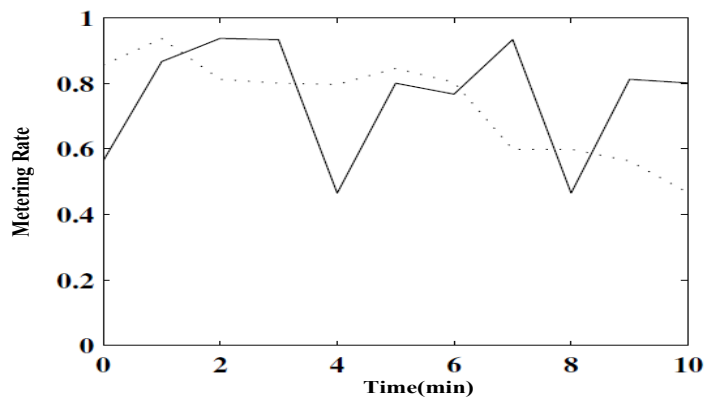

Fig. 6. The speed limits and ramp metering rates for controlled case

The MPC approach can predict the presence of congestion and prevent it or reduce its negative effects due to the deceleration of vehicles (using the speed limit), or delayed vehicles before they reach the congested area. 
When controlling group of vehicles by the full automation, able to maintain a small distance between the vehicles, which leads to the decrease in TTS.

\section{Conclusion}

It is showed that the model predictive control (MPC) can be used to determine the optimal speed of group of vehicles within the IVHS. The proposed approach was illustrated by practical examples based on modeling with the establishment of dynamic speed limits as control measures. These results show the potential benefits and the possibility of improvement that can be obtained using an intelligent control for MPC in IVHS.

\section{References}

1. Akopyan R.A., Makarov V.V. To an estimation of stability of movement of the car // Automobile Industry. 1976. № 3, pp. 23-25.

2. Akhmedov A.A. Improvement of controllability and stability of the car when driving on an uneven road using multicriterion parametric optimization methods. Diss. Cand. Tech. Sciences: 05.05.03. Moscow, 2004. 168 p.

3. Bakhmutov S.V., Bogomolov C.B. Increase of indicators of controllability and stability of the car by a method of multicriteria optimization // Proceedings of XV conference 'Active safety of the car'. Dmitrov: NITSIAMT, 1996.

4. Audet C. and J.E. Dennis Jr., Analysis of generalized pattern searches, SIAM Journal on Optimization, vol. 13, no. 3, pp. 889-903, 2007.

5. Brackstone M. and McDonald M. "Car-following: A historical review," Transportation Research Part F, vol. 2, no. 4, pp. 181-196, 1999.

6. A May. Traffic Flow Fundamentals. Englewood Cliffs, New Jersey: Prentice-Hall, 1990.

7. Kometani E. and Sasaki T. "Dynamic behaviour of traffic with a nonlinear spacing speed relationship," in Proceedings of the Symposium for Theory Traffic Flow, Research Laboratories, General Motors, New York, 1959, pp. 105-109.

8. Michaels R.M. "Perceptual factors in car following," in Proceedings of the 2nd International Symposium for Theory Road Traffic Flow, Paris, France, 1963, pp. 44-59.

9. Nagel K. "Particle hopping models and traffic flow theory," Physical Review E, vol. 53, pp. 4655-4672, 1996. 
10. Gazis D., Herman R., and Rothery R. "Nonlinear follow leader the models of traffic flow," Operations Research, vol. 9, no. 4, pp. 545-567, June 1961.

11. Baskar L., B. De Schutter, and Hellendoorn H. "Hierarchical traffic control and management with intelligent vehicles," in Proceedings of the 2007 IEEE Intelligent Vehicles Symposium (IV'07), Istanbul, Turkey, June 2007, pp. 834-839.

\section{DATA ABOUT THE AUTHORS}

Nikolaev Andrey Borisovich, Honoris Causa, Doctor of Technical Sciences, Professor, Head of Department

State Technical University - MADI

64, Leningradsky prospekt, Moscow, 125319, Russian Federation nikolaev.madi@mail.ru

Myo Min Khaing, Postgraduate Student, Department of Automated Control Systems

State Technical University - MADI

64, Leningradsky prospekt, Moscow, 125319, Russian Federation myominkhain52@mail.ru

Aung Myo Thwin, Postgraduate Student, Department of Automated Control Systems

State Technical University - MADI

64, Leningradsky prospekt, Moscow, 125319, Russian Federation aungmyothwin252@gmail.com

Moe Ko Ko, Postgraduate Student, Department of Automated Control Systems

State Technical University - MADI

64, Leningradsky prospekt, Moscow, 125319, Russian Federation moekoko88@gmail.com

Myo Lin Aung, Postgraduate Student, Department of Automated Control Systems

State Technical University - MADI

64, Leningradsky prospekt, Moscow, 125319, Russian Federation 109miketee110@gmail.com 